\title{
Comparative Study between Contralateral and Ipsilateral Approaches in Endovascular Treatment of High Superficial Femoral Artery Lesions
}

\author{
Mohamed Abd El-Hamid Abd El-Rahman ${ }^{1}$, Hany Abd El-Momen Abd El-Fattah ${ }^{1}$, Maged Mohamed Abd \\ El-Aziz Ghanem ${ }^{2}$, Mahmoud Mohamed Mohamed Metwally ${ }^{3}$, Mohamed Ibrahem Abdo Ibrahem ${ }^{1 *}$ \\ ${ }^{1}$ Department of Vascular Surgery, ${ }^{2}$ Department of Radiodiagnosis, \\ ${ }^{3}$ Department of Clinical Pathology, Faculty of Medicine - Al-Azhar University \\ *Correspondence author: Mohamed Ibrahem Abdo Ibrahem, Mobile: (+20) 01145711155, \\ E-mail: mohamed.ibrahem393@yahoo.com
}

\begin{abstract}
Background: peripheral arterial disease (PAD) is defined as a slowly progressing, occlusive vascular disease of the extremities primarily due to atherosclerosis which can involve vasculitis and thrombosis. The incidence of PAD is increasing worldwide due to an overall increase in diabetes, obesity, and other cardiovascular disorders.

Objective: the purpose of this study was to compare the outcomes, including the safety, of antegrade and retrograde puncture for SFA angioplasty access especially when lesion occurs in proximal part of SFA. The effect of these approaches upon procedure time and radiation dose was also assessed.

Patients and Methods: a group of 40 consecutive patients (19 men, 21 women) were randomized for antegrade puncture or retrograde puncture of the common femoral artery. Following retrograde puncture the guidewire was turned' and placed into the superficial femoral artery. The time for gaining access, screening time, radiation dose, and complications were recorded.

Results: there was no significant difference in the time to achieve arterial access between the two groups but the retrograde puncture required a substantially longer screening time and resulted in a higher radiation dose than antegrade puncture.

Conclusion: antegrade puncture is the standard means of obtaining access to the common femoral artery to carry out infrainguinal intervention. The antegrade approach has the advantage of permitting the use of shorter tools, and additional support for manipulating catheters and guidewires.
\end{abstract}

Keywords: Endovascular treatment, PAD, SFA.

\section{INTRODUCTION}

Peripheral arterial disease (PAD) is defined as a slowly progressing, occlusive vascular disease of the extremities primarily due to atherosclerosis which can involve vasculitis and thrombosis. The incidence of PAD is increasing worldwide due to an overall increase in diabetes, obesity, and other cardiovascular disorders ${ }^{(\mathbf{1})}$.

The 2005 ACC/AHA guidelines on PAD posited that 5-year outcomes on limb morbidity of PAD patients is $70-80 \%$ having stable claudication or walking pain, $10-20 \%$ having worsening claudication or resting pain, and 1-2\% evolving to critical limb ischemia (CLI). Patients with resting pain and CLI have two to four times increased chance of coronary arterial disease and cerebrovascular disease with increased mortality ${ }^{(2)}$.

Peripheral arterial disease (PAD) of the superficial femoral artery (SFA) is the most common cause of intermittent claudication. Atherosclerotic disease of the SFA is localized to the region of Hunter s canal. An isolated occlusion or stenosis of the SFA often results in decreased perfusion of the leg, resulting in demand related, reversible, ischemic pain localized to the calf. Ischemic rest pain and tissue loss, also known as critical limb ischemia (CLI), are uncommon manifestations of isolated SFA disease. CLI is more commonly observed when occlusive disease of the SFA is combined with occlusive disease involving the below knee popliteal artery or tibial arteries ${ }^{(3)}$.
Subsequently, Gruentzig popularized the concept of catheter directed balloon angioplasty. Angioplasty disrupts the atherosclerotic plaque by displacing it radially. This action results in stretching of the adventitia thereby increasing the lumen diameter in the treated vessel ${ }^{(4)}$.

By definition, a dissection is created and if significant, can be flow limiting. Currently, the most commonly utilized endovascular revascularization options are percutaneous transluminal angioplasty (PTA) with provisional stenting or primary stenting. Provisional or selective stenting is indicated for the treatment of flow limiting dissections and/or persistent, hemodynamically significant stenosis or recoil after PTA. This approach is recommended by the Trans-Atlantic inter-Society Consensus document II (TASC II) when treating SFA disease. However, both PTA alone and primary stenting can successfully treat SFA disease. Therefore, the debate continues as to which endovascular treatment is superior ${ }^{(4)}$.

The superficial femoral artery (SFA) has long presented endovascular specialists with a unique challenge. This vessel is subjected to various forces, such as compression, torsion, flexion, extension, and contraction, which caused many treatments to fail. However, maintaining patency in the SFA was also crucial for preserving patients' quality of life and limbs ${ }^{(5)}$.

Numerous modalities now exist for treating the SFA. Self-expanding stents, covered stent grafts, 
angioplasty balloons, plaque atherectomy devices, and a variety of chronic total occlusion devices all provide endovascular specialists with a wide array of options. The question for many specialists is which tool in their vast armamentarium should they use on particular patients and lesions? Does one device treat all lesions best, or are other factors better indicators of which technology to use ${ }^{(5)}$.

Subintimal angioplasty (SA) was found to be an effective method for the treatment of long superficial femoral artery (SFA) occlusions. In this technique, a wire is advanced across the occlusion in the subintimal space, and then it re-enters the true vessel lumen distal to the occlusion. One of the major factors that limit the success of this procedure is the failure of the wire to re-enter the true lumen. This issue is often related to the presence of severe calcification in the vessel wall. In such cases, a retrograde popliteal approach can serve as a potential backup option ${ }^{\left({ }^{6}\right.}$.

\section{AIM OF THE WORK}

The aim of this work is to compare between contralateral and ipsilateral approaches in endovascular treatment of high superficial femoral artery lesions (stenosis or occlusion) in patients with critical ischemia or chronic lower limb ischemia having disabling claudication pain interfering with their social activities after failed medical treatment, as regard to the effectiveness and results of each procedure as well as complications.

\section{PATIENT AND METHODS}

\section{Patients:}

This is a prospective randomized study which was conducted at Al-Azhar University Hospitals. It was including forty patients who were admitted to the vascular surgery departments of Al-Azhar University Hospitals. This study was conducted a period from November 2017 to November 2018.

These forty patients were divided in to $\mathbf{2}$ main groups:-

Group A: This group including 20 patients with high superficial femoral artery lesion (stenosis or occlusion). The approach site of this access is contralateral femoral artery (contralateral approach).

Group B: This group including also 20 patients with high superficial femoral artery lesion (stenosis or occlusion). The approach site of this access is ipsilateral antegrade femoral artery (ipsilateral approach).

\section{Written informed consent:}

An approval of the study was obtained from AlAzhar University Academic and Ethical Committee. Every patient signed an informed written consent for acceptance of the operation.

Methods:
Randomized case-control study enrolling 40 patients randomly divided into two groups. Group A (access site from contralateral femoral artery) and group B (access site from ipsilateral femoral artery).

Full records: all patients' data were recorded according to the data supplied in the vascular sheet.

Examination: in the form of: 1- General examination and 2- Local examination

\section{Investigation:}

1- Laboratory.

2- Radiological:

A. $\mathrm{X}$ ray foot: if there was foot ulceration or gangrene, to detect osteomyelitis.

B. Duplex ultrasound: with assessment of (PSV)

C. Computed tomography angiography (CTA): recommended to be prior to standard conventional angiography as less invasive maneuver.

D. Conventional angiography: it was preserved for the pre intervention.

\section{Technique:}

Every case was studied individually and according to the mentioned criteria it was subjected to angioplasty, the procedure was done in the angio suite under complete aseptic technique. We used non-ionic contrast medium (ultravist). At the end of each procedure the details of technique was documented individually regarding:

*Anesthesia: local with or without IV sedation

* Site of arterial puncture:

Contralateral Procedure:

1 -Access was made by puncturing the common femoral artery at the contralateral side using the Seldinger technique through US guidance.

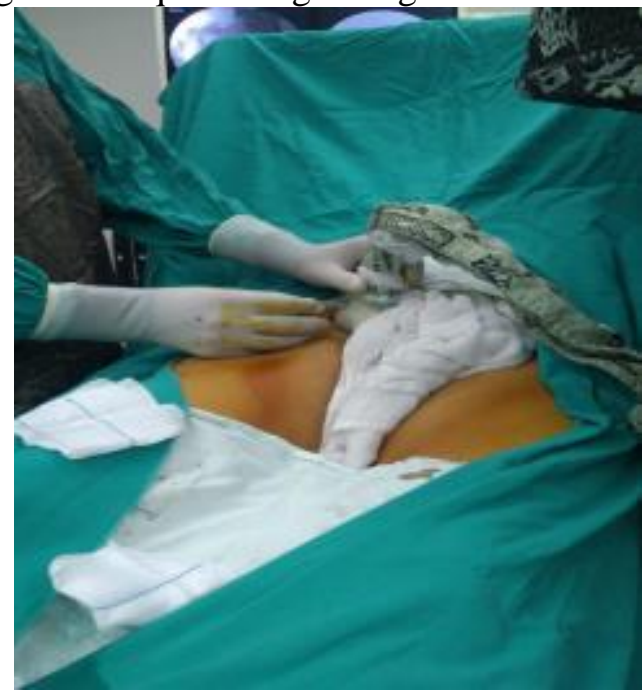

2- A short $(11 \mathrm{~cm}) 6 \mathrm{~F}$ straight sheath was installed at the puncture site.

3- Bifurcation crossing was performed with a 0.035 hydrophilic guidewire of $260 \mathrm{~cm}$, which was advanced into the aorta with a crossover catheter of choice of 65 or $100 \mathrm{~cm}$ (e.g., RIM, Simmons 1, Simmons 2, Universal Flush, Berenstein). 


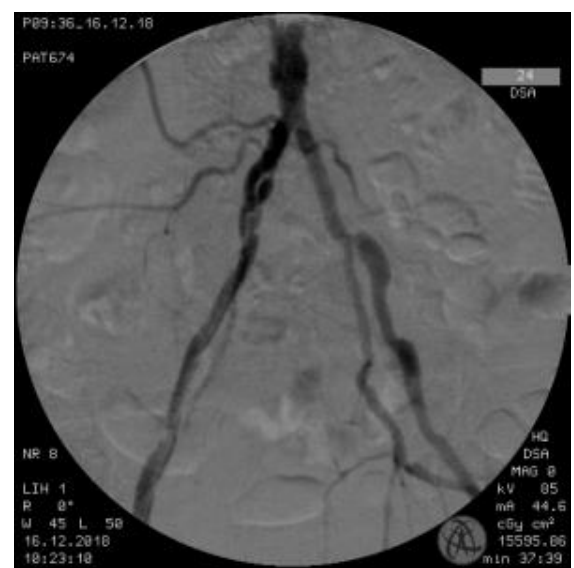

4- Crossover catheter is placed in the common femoral artery on the lesion side and over a stiffer guidewire (e.g., Bent Terumo, Amplatz,...)

5- Replacement of short sheath by A long 4F-6F sheath (cross over) was installed in the common femoral artery.

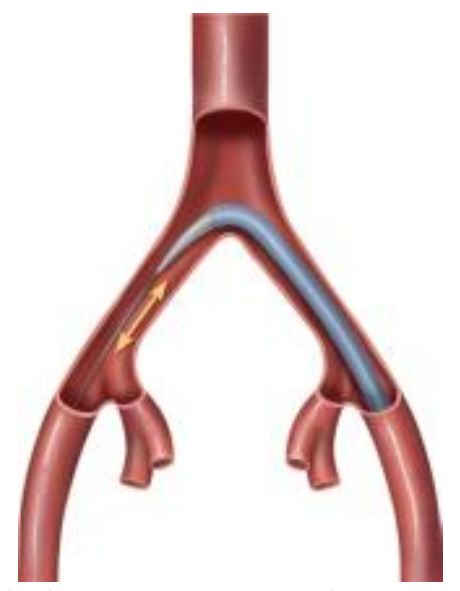

6- Intraluminal lesion passage was done by advancing the hydrophilic guide wire and crossover catheter into the superficial femoral artery (SFA) of the contralateral limb.

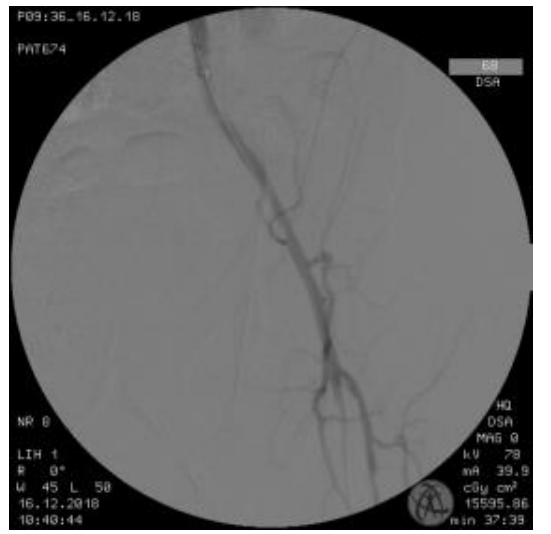

7- Intraluminal lesion passage was performed with the catheter (of choice) over the guide wire.

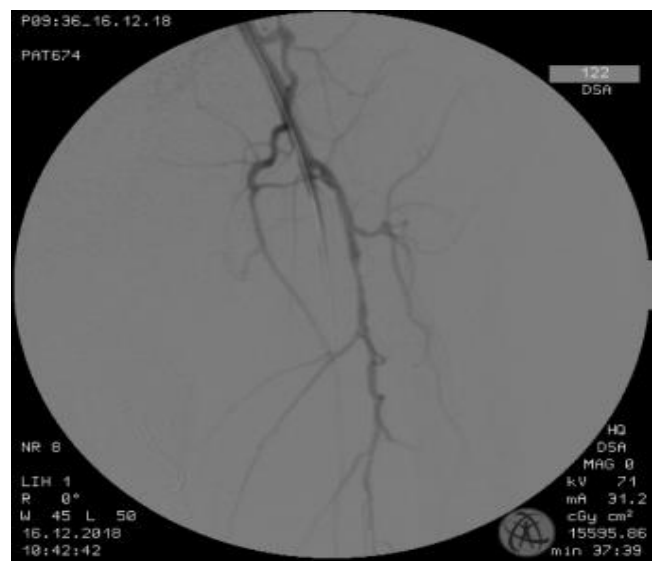

9- Lesion treatment was done by means of (a) PTA

(b) Stent placement either

. Flexible self-expanding nitinol

. Long self-expanding nitinol

. Drug-eluting self-expanding nitinol

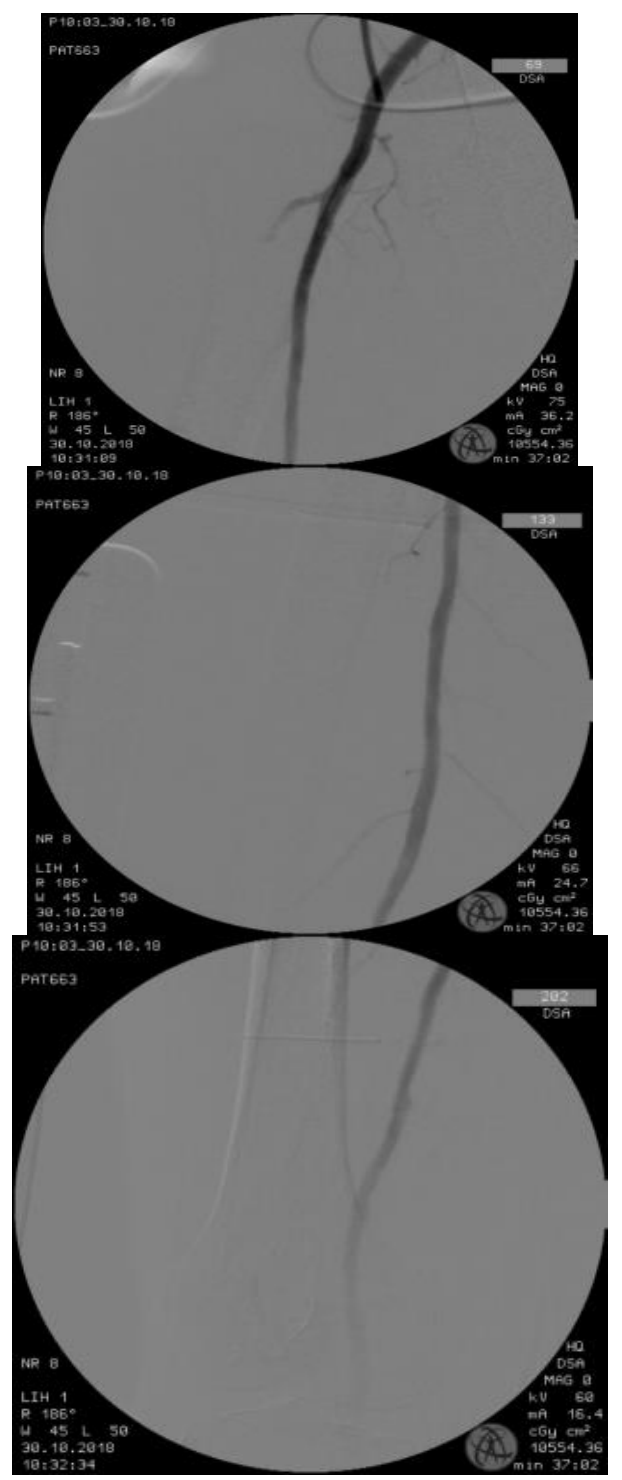

10- A completion angiography was performed. If the result was insufficient after PTA (residual stenosis $30 \%$ or fl ow-limiting dissection), a prolonged balloon dilation was performed. When the result remain unsatisfying, stent placement was done. 


\section{Ipsilateral Procedure}

1. Access is made by puncturing the common femoral artery at the ipsilateral side using the Seldinger technique. The entrance into the common femoral artery should be in the upper half of the femoral head. If not the wire should invariably go into the profunda femoris/deep femoral artery. In this event it was almost invariably better to remove the needle and hold pressure for a few minutes. Subsequently a reattempt at a higher level should be done.

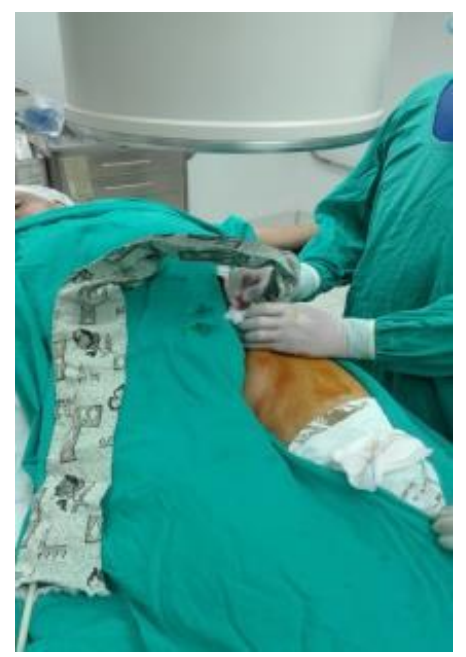

2. The remaining steps were the same as for contralateral access with the exception that crossover did not have to be achieved.

\section{Statistical analysis}

Recorded data were analyzed using the statistical package for social sciences, version 20.0 (SPSS Inc., Chicago, Illinois, USA). Quantitative data were expressed as mean \pm standard deviation (SD). Qualitative data were expressed as frequency and percentage.

\section{The following tests were done:}

- Independent-samples t-test of significance was used when comparing between two means.

- Chi-square $\left(\mathrm{x}^{2}\right)$ test of significance was used in order to compare proportions between two qualitative parameters.

- The confidence interval was set to $95 \%$ and the margin of error accepted was set to $5 \%$. The p-value was considered significant as the following:

- Probability (P-value)

- P-value <0.05 was considered significant.

- P-value $<0.001$ was considered as highly significant.

- P-value >0.05 was considered insignificant.

\section{RESULTS}

I-Demographic and Clinical Characteristics:

The present study was conducted on 40 patients, 19 males $(47.5 \%)$ and 21 females $(52.5 \%)$.
Table 1: Distribution of the studied patients regarding their sex.

\begin{tabular}{|l|l|l|l|l|}
\hline & \multicolumn{2}{|l|}{ Contralateral } & \multicolumn{2}{l|}{$\begin{array}{l}\text { Ipsilateral } \\
\text { approaches }\end{array}$} \\
\hline Sex & N. & $(\%)$ & N. & $(\%)$ \\
\hline Male & 9 & $45 \%$ & 10 & $50 \%$ \\
\hline Female & 11 & $55 \%$ & 10 & $50 \%$ \\
\hline p-value & $>0.05$ & \multicolumn{3}{|l}{} \\
\hline
\end{tabular}

Their age ranged between 50 years and 87 years with a mean age of $66.7 \pm 12.35$.

Table 2: Distribution of the studied patients regarding their age.

\begin{tabular}{|l|l|l|c|}
\hline & Contralateral & $\begin{array}{c}\text { Ipsilateral } \\
\text { approaches }\end{array}$ & $\begin{array}{c}\text { p- } \\
\text { value }\end{array}$ \\
\hline $\begin{array}{l}\text { Age } \\
\text { (Mean } \pm \text { S.D) }\end{array}$ & $58.2 \pm 12.33$ & $57.2 \pm 11.24$ & $\begin{array}{c}> \\
0.05\end{array}$ \\
\hline
\end{tabular}

The associated comorbidities were IHD and C.V.S. The associated risk factors were D.M., smoking, hypertension and dyslipidemia.

Table 3: The associated diseases and risk factors in the studied patients

\begin{tabular}{|l|l|c|}
\hline & Contralateral & $\begin{array}{c}\text { Ipsilateral } \\
\text { approaches }\end{array}$ \\
\hline IHD & 4 & 3 \\
\hline C.V.S. & 3 & 4 \\
\hline D.M. & 13 & 12 \\
\hline Smoking & 9 & 10 \\
\hline Hypertension & 8 & 11 \\
\hline Dyslipidemia & 12 & 11 \\
\hline p-value & \multicolumn{2}{|c}{$\mathbf{0 . 0 5}$} \\
\hline
\end{tabular}

Symptoms and signs of patients included in this study included rest pain, ischemic ulcers, gangrene of toe, heel or forefoot.

Table 4: Symptoms and signs of patients included in this study

\begin{tabular}{|l|l|l|}
\hline $\begin{array}{l}\text { Symptoms and } \\
\text { signs }\end{array}$ & Contralateral & $\begin{array}{l}\text { Ipsilateral } \\
\text { approaches }\end{array}$ \\
\hline Rest pain & 4 & 5 \\
\hline $\begin{array}{l}\text { Ischemic } \\
\text { ulcers }\end{array}$ & 6 & 7 \\
\hline Gangrene: & 10 & 9 \\
Toe & 4 & 3 \\
Heel & 3 & 2 \\
Forefoot & 3 & 4 \\
\hline
\end{tabular}


III-Procedure (Technical) outcome:

Success rate:

Table 5: Success rate results

\begin{tabular}{|c|c|c|c|c|c|c|c|c|c|c|c|c|c|c|c|c|}
\hline & \multicolumn{8}{|c|}{ Contralateral approach } & \multicolumn{8}{|c|}{ Ipsilateral approach } \\
\hline & \multicolumn{4}{|c|}{ Stenosis } & \multicolumn{4}{|c|}{ Total occlusion } & \multicolumn{4}{|c|}{ Stenosis } & \multicolumn{4}{|c|}{ Total occlusion } \\
\hline & \multicolumn{2}{|c|}{$\begin{array}{l}\text { Flush } \\
\text { Lesion }\end{array}$} & \multicolumn{2}{|c|}{$\begin{array}{c}\text { Proximal } \\
1 / 3\end{array}$} & \multicolumn{2}{|c|}{$\begin{array}{l}\text { Flush } \\
\text { Lesion }\end{array}$} & \multicolumn{2}{|c|}{\begin{tabular}{|c|} 
Proximal \\
$1 / 3$
\end{tabular}} & \multicolumn{2}{|r|}{$\begin{array}{l}\text { Flush } \\
\text { Lesion }\end{array}$} & \multicolumn{2}{|c|}{$\begin{array}{c}\text { Proximal } \\
1 / 3\end{array}$} & \multicolumn{2}{|c|}{$\begin{array}{l}\text { Flush } \\
\text { Lesion }\end{array}$} & \multicolumn{2}{|c|}{\begin{tabular}{|c|} 
Proximal \\
$1 / 3$
\end{tabular}} \\
\hline & $\mathrm{N}$ & $\%$ & $\mathrm{~N}$ & $\%$ & $\mathrm{~N}$ & $\%$ & $\mathrm{~N}$ & $\%$ & $\mathrm{~N}$ & $\%$ & $\mathrm{~N}$ & $\%$ & $\mathrm{~N}$ & $\%$ & $\mathrm{~N}$ & $\%$ \\
\hline Failed & 2 & $22.2 \%$ & 0 & $0.0 \%$ & 1 & $25.0 \%$ & 1 & $33.3 \%$ & 2 & $22.2 \%$ & 1 & $20.0 \%$ & 3 & $75.0 \%$ & 1 & $50.0 \%$ \\
\hline Successful & 7 & $77.8 \%$ & 4 & $100.0 \%$ & 3 & $75.0 \%$ & 2 & $66.7 \%$ & 7 & $77.8 \%$ & 4 & $80.0 \%$ & 1 & $25.0 \%$ & 1 & $50.0 \%$ \\
\hline
\end{tabular}

The early patency rate was $80 \%$ in the group with contralateral angioplasty, and $65 \%$ in the group with ipsilateral angioplasty.

Table 6: Technical outcome results

\begin{tabular}{||l|l|l|l|l|}
\hline \multirow{2}{*}{} & \multicolumn{2}{|c|}{ Contralateral } & \multicolumn{2}{c|}{ Ipsilateral } \\
\cline { 2 - 5 } & \multicolumn{1}{|c|}{$\mathrm{N}$} & $\%$ & $\mathrm{~N}$ & $\%$ \\
\hline Failed & 4 & $20.0 \%$ & 7 & $35.0 \%$ \\
\hline Successful (patent) & 16 & $80.0 \%$ & 13 & $65.0 \%$ \\
\hline P-value & $>0.05$ & \multicolumn{3}{|l}{} \\
\hline
\end{tabular}

\section{IV-Clinical outcome:}

Early results including limb salvage and amputation rate were followed postoperatively.

These findings were shown together between contralateral technique' and ipsilateral techniques in table 7 .

Limb salvage and amputation rate are shown in table 7. Two early major amputations were done in a contralateral group (6.6\%). In a group with ipsilateral approach amputation rate was done in $(11.60 \%)$.

The early limb salvage rate was $93.67 \%$ in the group with contralateral technique.

Table 7: Early outcome results

\begin{tabular}{|l|l|l|}
\hline Early results & Contralateral & Ipsilateral approaches \\
\hline Limb salvage & $93.67 \%$ & $86.33 \%$ \\
\hline Amputation & $6.60 \%$ & $11.60 \%$ \\
\hline
\end{tabular}

Late results at three months postoperatively are shown in table 8 . The late limb salvage rate in the group with contralateral angioplasty technique was $66.67 \%$ while in the group with ipsilateral technique $73.33 \%$.

Table 8: Late results (At three months postoperatively)

\begin{tabular}{|l|l|l|}
\hline Late results & Contralateral & Ipsilateral approaches \\
\hline Redo operations & $9.09 \%$ & $3.125 \%$ \\
\hline Limb salvage & $75.41 \%$ & $76.05 \%$ \\
\hline Amputation & $24.59 \%$ & $23.95 \%$ \\
\hline
\end{tabular}

Limb salvage was defined as freedom from major amputation. Toe, ray, or transmetatarsal amputations were considered as minor amputations.

Clinical success were defined as relief of rest pain or improve healing of the ulcer and limb salvage, based on Rutherford categories.

\section{V- Post-procedural evaluation:}

\section{a- Ankle brachial pressure index (ABPI) evaluation:}

The pre-intervention mean ABPI for the 40 patients was $0.29 \pm 0.2$, which improved to $0.58 \pm 0.17$ immediately post-intervention $(\mathrm{P}<0.001)$. When evaluated after 1 months post-procedure, it improved to $0.62 \pm 0.19(\mathrm{P}<0.001)$.

Table 9: Ankle brachial pressure index (ABPI) in the studied patients group pre- and postoperative.

\begin{tabular}{|l|c|c|c|c|}
\hline & Pre intervention & $\begin{array}{c}\text { Immediately after } \\
\text { angioplasty }\end{array}$ & $\begin{array}{c}\text { After three months post } \\
\text { angioplasty }\end{array}$ & $\mathrm{P}$ \\
\hline Contralateral & $0.29 \pm 0.3$ & $0.57 \pm 0.19$ & $0.62 \pm 0.14$ & $<0.001$ \\
\hline Ipsilateral & $0.29 \pm 0.2$ & $0.58 \pm 0.17$ & $0.63 \pm 0.28$ & $<0.001$ \\
\hline
\end{tabular}




\section{b- Duplex scan evaluation:}

There was statistically significant improvement in the type of wave pattern by duplex scan preoperative and postoperative (Table 10).

Table 10: Analysis of the relationship between pattern of waves pre and postoperative by colour duplex

\begin{tabular}{|l|l|l|}
\hline Pattern of the waves & Contralateral & Ipsilateral approaches \\
\hline Monophasic & 4 & 7 \\
\hline Biphasic & 10 & 9 \\
\hline Triphasic & 6 & 4 \\
\hline P value & $<0.05$ & \\
\hline
\end{tabular}

\section{VI- Complications}

Table 11 shows perioperative complications

Table 11: Perioperative complications

\begin{tabular}{|c|c|c|c|c|c|}
\hline & \multicolumn{2}{|c|}{ Contralateral } & \multicolumn{2}{|c|}{ Ipsilateral approach } \\
\hline & & Count & $\%$ & Count & $\%$ \\
\hline \multirow{8}{*}{ Complications } & Femoral pseudoaneurysm & 0 & $0.0 \%$ & 1 & $5.0 \%$ \\
\hline & Groin hematoma & 0 & $0.0 \%$ & 2 & $10.0 \%$ \\
\hline & Retroperitoneal hematoma & 0 & $0.0 \%$ & 1 & $5.0 \%$ \\
\hline & Groin infection & 1 & $5.0 \%$ & 1 & $5.0 \%$ \\
\hline & HTN Pulmonary edema & 1 & $5.0 \%$ & 0 & $0.0 \%$ \\
\hline & Scrotal hematoma & 0 & $0.0 \%$ & 1 & $5.0 \%$ \\
\hline & SFA dissection & 0 & $0.0 \%$ & 1 & $5.0 \%$ \\
\hline & No complication & 18 & $90.0 \%$ & 13 & $65.0 \%$ \\
\hline
\end{tabular}

Table 12: Perioperative complications

\begin{tabular}{|l|c|c|c|c|}
\hline \multirow{2}{*}{} & \multicolumn{2}{|c|}{ Contralateral } & \multicolumn{2}{c|}{ Ipsilateral approach } \\
\cline { 2 - 5 } & Count & $\%$ & Count & $\%$ \\
\hline No complication & 18 & $90.0 \%$ & 13 & $65.0 \%$ \\
\hline complication & 2 & $10 \%$ & 7 & $35 \%$ \\
\hline p-value & \multicolumn{4}{|c|}{$>0.05$} \\
\hline
\end{tabular}

\section{VII- Mortality:}

Mortality occurred in one patients due to myocardial infarction. No patients were lost during the period of follow-up. Mortality was not related to the procedure, but was attributed to the associated comorbidities.

\section{DISCUSSION}

In this study we have compared retrograde and antegrade arterial puncture for gaining femoral access in patients requiring proximal superficial femoral artery intervention. Antegrade puncture is the standard means of obtaining access to the common femoral artery to carry out infrainguinal intervention. This access requires a much higher puncture than usual with a longer subcutaneous tract compromised by the abdominal apron, particularly in obese patients and therefore is technically more difficult. If a sufficiently shallow angle of puncture of the common femoral artery is not obtained then the guidewire preferentially enters the profunda femoris artery requiring additional wire and catheter manipulation and prolonging the procedure ${ }^{(7)}$.
It is important to notice that presence of lesion in proximal part of SFA needs suprainguinal puncture of the iliac artery which is better to be avoided as it cannot be effectively compressed. A report of the National Confidential Enquiry into Perioperative deaths stating that "Cannulation of the femoral artery should always be below the inguinal ligament to avoid the danger of retro-peritoneal hematoma" stimulated discussion about the role of puncture site in hematoma formation. ${ }^{(8)}$.

Retrograde punctures are technically easier to perform and most operators have much greater experience with them as they are routinely used for obtaining access for diagnostic angiography. The puncture tracts, except in very obese patients, are usually short. In the absence of severe aorto-iliac disease, turning the catheter into the ipsilateral iliac system and then into the SFA is relatively straightforward and takes a short time to learn ${ }^{(7)}$.

In our study the skin puncture site is lower than the abdominal apron and is therefore easier to palpate with less likelihood of suprainguinal puncture. Although screening is required in all patients, thus 
giving a higher average of radiation dose than in antegrade punctures, manipulation of the guidewire and catheter down the SFA is relatively straightforward.

As retrograde punctures are often easier to perform, particularly in obese patients, we felt that this approach may potentially reduce the complication rates associated with infra-inguinal intervention these data are similar to data collected from ${ }^{(9)}$.

Although in this small study we have shown that there were fewer immediate complications with the retrograde group, probably because of the fewer punctures that were required, there were no major safety differences between the two techniques. The complications that resulted were minor, not requiring treatment or delay in discharge.

Conclusions regarding any differences between the incidences of major complications cannot be drawn without a larger subject group.

In our study there was no significant difference in the time to achieve arterial access between the two groups but the retrograde puncture required a substantially longer screening time and resulted in a higher radiation dose than antegrade puncture. This is due to the necessity of screening of the catheter and wire down from the aorta to the femoral artery in all patients in the retrograde group, through the thickest part of the patient, i.e., abdomen and pelvis. These data are similar to data collected from ${ }^{(9)}$.

In our study because of the proposed difficulty encountered with antegrade punctures, particularly in obese patients, we wished to see if retrograde punctures offered any advantages. The antegrade approach has the advantage of permitting the use of shorter tools, and additional support for manipulating catheters and guidewires.

Its main drawbacks are the more demanding technical skills required for percutaneous puncture of the CFA and the potential difficulties of entering the SFA, avoiding its origin. Ultrasound guided puncture may help to increase the success rate, especially in obese patients (10). Antegrade access is especially difficult if a stenosis in the CFA is present or stenosis or occlusion of proximal SFA is also present.

The contralateral crossover approach is preferred by many vascular surgeons, in order to gain distance to the target lesion and allow a more comfortable working area, as well as minimising the risk of hematoma and the need for compression on the ipsilateral groin.

However, the use of longer devices within tortuous iliac arteries may prove a major inconvenience for a correct navigation and deployment of larger devices Theses data are similar to data collected from ${ }^{(\mathbf{1 0})}$.

\section{CONCLUSION}

Antegrade puncture is the standard means of obtaining access to the common femoral artery to carry out infrainguinal intervention.

The antegrade approach has the advantage of permitting the use of shorter tools, and additional support for manipulating catheters and guidewires.

It is important to notice that presence of lesion in proximal part of SFA needs suprainguinal puncture of the iliac artery which is better to be avoided as it cannot be effectively compressed.

Retrograde punctures are technically easier to perform and most operators have much greater experience with them as they are routinely used for obtaining access for diagnostic angiography.

The contralateral crossover approach is preferred by many vascular surgeons, in order to gain distance to the target lesion and allow a more comfortable working area, as well as minimising the risk of hematoma and the need for compression on the ipsilateral groin.

\section{REFERENCES}

1. Pattillo CB, Bir S, Rajaram $V$ et al. (2011): Inorganic nitrite and chronic tissue ischaemia: a novel therapeutic modality for peripheral vascular diseases. Cardiovascular Research, 89: 533-41.

2. Flu H, van der Hage, Knippenberg JH et al. (2008): Treatment for peripheral arterial obstructive disease: An appraisal of the economic outcome of complications. Journal of Vascular Surgery, 48: 368-76.

3. Tadros RO, Vouyouka AG, Ting W et al. (2015) A Review of Superficial Femoral Artery Angioplasty and Stenting. J Vasc Med Surg., 3: 183.

4. Norgren L, Hiatt $W$, Dormandy $J$ et al. (2014): Inter Society Consensus for the Management of Peripheral Arterial Disease (TASC II). Eur J Vasc Endovasc Surg., 33:1-75

5. Cote AV, Berger PB, Holmes DR et al. (2011): Hemorrhagic and vascular complications after percutaneous coronary intervention with adjunctive abciximab. Mayo Clin Proc., 76:890-896.

6. Ko YG, Kim JS, Choi DH et al. (2007): Improved technical success and midterm patency with subintimal angioplasty compared to intraluminal angioplasty in long femoropopliteal occlusions. J Endovasc Ther., 14:374381.

7. Grenon SM, Reilly LM, Ramaiah VG (2011): Technical endovascular highlights for crossing the difficult aortic bifurcation. J Vasc Surg., 54:89-96.

8. Whimster F, Raphael M, Hartnell G (2001): Femoral artery catheterisation and retroperitoneal haematoma formation (Letter). Clin Radiol., 56:933-934.

9. Nice C, Timmons G, Bartholemew P et al. (2013): Retrograde vs. Antegrade puncture for infra-inguinal angioplasty. Cardiovasc Intervent Radiol., 26:370 -374.

10. Miralles M, Candela E, Blanes E et al. (2016): Reverse Retrograde Approach: An Alternative Method for Ipsilateral Access to the Superficial Femoral Artery EJVES Short Reports, https://www.ejvesreports.com/article/S24056553(15)00023-7/fulltext 